\title{
Azaperone e sua associação com xilazina ou dexmedetomidina em suínos
}

\author{
Azaperone and its association with xilazina or dexmedetomidina in swines
}

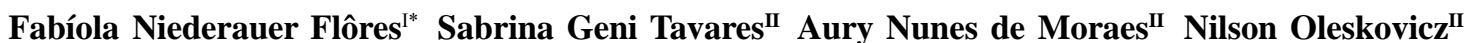 \\ Luiz César Pereira Santos ${ }^{\text {II }}$ Vanessa Minsky ${ }^{\text {II }}$ Emília Keshen ${ }^{\text {II }}$
}

\section{RESUMO}

\begin{abstract}
$O$ estudo objetivou avaliar o efeito sedativo do azaperone e de sua associação com a xilazina ou dexmedetomidina na espécie suína, assim como verificar a possibilidade de o agente butirofenônico contrabalançar os efeitos causados pelos agonistas $\alpha_{2}$-adrenérgicos nos parâmetros cardiovasculares. Foram estudados 18 suínos hígidos da linhagem Dambread XMS 50, de 50 dias de idade, pesando $17,3 \mathrm{~kg}( \pm 1,7)$. Todos os animais foram submetidos a anestesia com isofluorano para instrumentação necessária ao protocolo experimental e, 30 minutos após a recuperação anestésicas, os parâmetros basais foram mensurados e os animais alocados aleatoriamente em três grupos de seis animais cada: GA (Azaperone $2 \mathrm{mg} \mathrm{kg}^{-1}+$ Cloreto de sódio 0,5ml - IM), GAD (Azaperone $2 \mathrm{mg} \mathrm{kg}^{-1}+$ Dexmedetomidina $3 \mu \mathrm{g} \mathrm{kg}^{-1}-\mathrm{IM}$ ), GAX (Azaperone $2 \mathrm{mg} \mathrm{kg}^{-1}+$ Xilazina $2 \mathrm{mg} \mathrm{kg}^{-1}$ - IM). Os parâmetros foram novamente avaliados aos 15, 30, 45 e 60 minutos após administração dos fármacos correspondentes aos grupos do estudo. A frequência cardiaca teve seus valores reduzidos em todos os grupos, porém essa redução foi maior no GAX. Durante o estudo não foi observado efeito bifásico sobre a pressão arterial, com hipertensão seguida de hipotensão. OGAX apresentou redução de $\mathrm{PaO}_{2} e$ aumento de $\mathrm{PaCO}_{2}$, assim como observou-se melhor efeito sedativo nesse grupo. Os resultados permitem concluir que a associação de azaperone com xilazina promoveu melhor sedação e miorrelaxamento e menor resposta a estímulos.
\end{abstract}

Palavras-chave: azaperone, xilazina, dexmedetomidina, suinos.

\section{ABSTRACT}

The aim of this study was to evaluate the sedative effects of azaperone and its association with xylazine or dexmedetomidine in swine, as well as verifying the possibility of the butyrophenone agent to counterbalance the effects caused by $\alpha_{2}$-adreneceptor agonists on the cardiovascular and hemodynamic parameters. For this, eighteen healthy swines of the Dambread X MS 50 lineage aged 50 days-old, weighing around $17.3 \mathrm{~kg}( \pm 1.7)$ were used. All animals were submitted an isoflurane anesthesia by face mask throughout the period of instrumentation. Basal parameters were measured 30 minutes after recovering from general anesthesia. All swines were randomly assigned into three groups of six animals each: $A G$ (azaperone $2 \mathrm{mg} \mathrm{kg}^{-1}+$ sodium chloride $0.5 \mathrm{ml}-\mathrm{IM}$ ), $A D G$ (azaperone $2 \mathrm{mg} \mathrm{kg}^{-1}+$ dexmedetomidine $3 \mu \mathrm{g} \mathrm{kg}^{-1}-\mathrm{IM}$ ) and AXG (azaperone $2 m g \mathrm{~kg}^{-1}+$ xylazine $2 \mathrm{mg} \mathrm{kg}^{-1}-\mathrm{IM}$ ). Parameters were again measured at the following times: 15, 30, 45 and 60 minutes after administrating the corresponding drugs to each group. The heart rate had its values reduced in all groups; however this reduction was more significative in AXG. During the study was not observed a biphasic effect over the arterial pressure with an initial increase followed by a gradual decrease. AXG presented reduction of $\mathrm{PaO}_{2}$ and an increase in $\mathrm{PaCO}_{2}$ as well as a better sedative effect. The results allow to conclude that the association of azaperone with xylazine promoted a better tranquilization and muscular relaxation.

Key words: azaperone, xylazine, dexmedetomidine, swines.

\section{INTRODUÇÃO}

A espécie suína (Sus scrofa domestica), reconhecida mundialmente pela sua importância na agropecuária mundial, é também considerada excelente modelo animal para experimentação. Dentre as espécies de animais domésticos, é a que apresenta maior

IUniversidade do Estado de Santa Catarina (UDESC), Hospital Veterinário. Av. Luiz de Camões, 2090, Bairro Conta Dinheiro, 88520-000, Lages, SC, Brasil. E-mail: fanf_vet@yahoo.com.br.*Autor para correspondência.

"UDESC, Lages, SC, Brasil. 
similaridade aos humanos, incluindo tamanho, estrutura e órgãos internos, padrões alimentares, enzimas gástricas e sistema endócrino. Todas essas características fazem com que essa espécie seja o modelo ideal para transplante de órgãos (CENTENO et al., 2004).

O azaperone é um neuroléptico da classe das butirofenonas muito utilizado em suínos, principalmente para introdução de novos animais em lotes (MUIR \& HUBBEL, 2001). Seu mecanismo de ação é similar ao das fenotiazínas, mimetizando a ação do ácido gama aminobutírico (GABA). Eles previnem a modulação do ácido glutâmico na junção sináptica do sistema extrapiramidal. Seus efeitos cardiovasculares sugerem um bloqueio periférico do a-adrenoceptor, muito similar aos fenotiazínicos (TRANQUILLI \& GRIMM, 1996), promovendo hipotensão, além de hiperventilação e diminuição nos valores de $\mathrm{PaCO}_{2}$ (ORR et al., 1976).

Os agentes agonistas $\alpha_{2}$-adrenérgicos, quando administrados em animais produzem sedação dose-dependente, analgesia, alterações significantes no sistema cardiovascular, como bradicardia, diminuição do débito cardíaco e hipertensão seguida de hipotensão, principalmente por estimulação de receptores centrais (MOENS, 2000; SINCLAIR, 2003).

A xilazina é amplamente utilizada em suínos (THURMON et al., 1996), embora o potente efeito sedativo que o fármaco apresenta nas demais espécies não seja observado na espécie suína, sendo portanto recomendado o emprego da xilazina em associação a outro agente sedativo ou anestésico para a obtenção de efeitos mais satisfatórios (BAUCK, 1984). A dexmedetomidina é um fármaco altamente seletivo para os receptores $\alpha_{2}$. Ela é um D-isômero 10 vezes mais específico que a xilazina, (SAVOLA \& SAVOLA., 1996) e sete vezes mais específico que a clonidina (TAKROURI et al., 2002).

O presente estudo tem por objetivos avaliar os efeitos sedativos e miorrelaxantes do azaperone isoladamente ou em associação à xilazina ou dexmedetomidina na espécie suína e avaliar a possibilidade de o azaperone amenizar o efeito de hipertensão produzido pelos agonistas $\alpha_{2}$ adrenérgicos.

\section{MATERIAL E MÉTODOS}

Foram empregados 18 suínos hígidos da linhagem Dambread X MS50 (Padrão Light), com idade média de 50 dias, machos ou fêmeas, pesando $17,3 \mathrm{~kg}$ $( \pm 1,7)$. Os animais foram submetidos a um período de ambientação de 15 dias, quando foram manejados pela equipe e transportados até a sala onde se realizaria o experimento a fim de minimizar o estresse no momento do procedimento.

Após jejum alimentar de 12 horas e hídrico de seis horas, os animais foram submetidos à anestesia geral inalatória com isofluorano ${ }^{\mathrm{a}}$ em vaporizador universal com fluxo de oxigênio de $30 \mathrm{a} 60 \mathrm{ml} \mathrm{kg}^{-1} \mathrm{~min}^{-1}$, por meio de máscara facial, para a instrumentação necessária ao estudo, quando foram realizadas a dissecação e canulação da artéria femoral com adaptação de sonda uretral heparinizada $\mathrm{n} \times 6$ acoplada a um agulha $40 \times 12$ e dispositivo adaptador PRN $^{\mathrm{b}}$, posteriormente suturados à pele do animal.

O protocolo experimental iniciou-se 30 minutos após a recuperação anestésica necessária ao procedimento de instrumentação, em sala com temperatura mantida em torno de $23,5^{\circ} \mathrm{C}$. Na seqüência, os animais foram aleatoriamente alocados em um dos seguintes grupos: GA ( $\mathrm{n}=06)$ : Azaperone ${ }^{\mathrm{c}}\left(2 \mathrm{mg} \mathrm{kg}^{-1}\right.$ $\mathrm{IM})+$ Cloreto de sódio $0,9 \%(0,5 \mathrm{ml}-\mathrm{IM}) ; \mathrm{GAD}(\mathrm{n}=06)$ : Azaperone (2mg kg-1 $-\mathrm{IM})+$ Dexmedetomidina $^{\mathrm{d}}(3 \mu \mathrm{g}$ $\mathrm{kg}^{-1}$ - IM); e GAX (n=06): Azaperone $\left(2 \mathrm{mg} \mathrm{kg}^{-1}-\mathrm{IM}\right)+$ Xilazina $^{\mathrm{e}}\left(2 \mathrm{mg} \mathrm{kg}^{-1}-\mathrm{IM}\right)$.

A administração dos fármacos foi realizada após a mensuração do tempo basal (T0), sendo realizada de forma simples cega, com os agentes aplicados em seringa única pela via IM na região cervical. Os tempos de avaliação dos parâmetros relacionados foram: valor basal T0: 30 minutos após a recuperação anestésica necessária para a instrumentação, quando posteriormente administraram-se os agentes correspondentes ao estudo e aos 15 (T15), 30 (T30), 45 (T45) e 60 (T60) minutos após T0.

Os parâmetros frequência cardíaca (FC), saturação de oxihemoglobina $\left(\mathrm{SpO}_{2}\right)$, temperatura retal (TR), temperatura cutênea (TC), avaliação invasiva de pressão arterial sistólica, média e diastólica (PAS, PAM e PAD) foram mensurados por meio de monitor multiparamétricof ${ }^{\mathrm{f}}$. A freqüência respiratória $(f)$ foi obtida por observação do movimento costo-abdominal com o animal posicionado em decúbito lateral. A coleta de sangue arterial para obtenção dos valores de pressão parcial de oxigênio $\left(\mathrm{PaO}_{2}\right)$, pressão parcial de dióxido de carbono $\left(\mathrm{PaCO}_{2}\right)$, potencial hidrogeniônico $(\mathrm{pH})$, potássio $\left(\mathrm{K}^{+}\right)$, sódio $\left(\mathrm{Na}^{+}\right)$, bicarbonato $\left(\mathrm{HCO}_{3}^{-}\right)$, hemoglobina $(\mathrm{Hb})$ e excesso de bases $(\mathrm{EB})$ foi realizada por meio da sonda inserida na artéria. As amostras $(0,5 \mathrm{ml})$ foram coletadas anaerobicamente em seringas heparinizadas e analisadas imediatamente. Aglicemia foi mensurada com o auxílio do aparelho portátil ${ }^{\mathrm{g}}$. Os dados não paramétricos de sedação, relaxamento muscular e resposta a estímulos sonoros foram avaliados por um único avaliador com base em escores 
estabelecidos, sendo: sedação, (0) sem sedação; (1) sedação leve; (2) sedação moderada; (3) sedação profunda; relaxamento muscular, (0) tônus normal; (1) tônus suave; (2) tônus moderado; (3) tônus exacerbado; resposta a estímulos, (0) resposta normal; (1) reage com os olhos e o corpo; (2) reage apenas com os olhos; (3) não reage.

Para avaliação estatística, foi empregado programa Sigma Stat for Windows Versão 3.0.1, SPSS Inc. 2003. Os dados paramétricos foram submetidos à análise de variância (ANOVA) de uma via com repetições múltiplas para avaliação entre grupos dentro de cada tempo. As diferenças encontradas foram submetidas ao Teste de Student Newman Keuls $(\mathrm{P} \leq 0,05)$. Para a análise entre tempos dentro de cada grupo, os dados foram submetidos à ANOVA de uma via e ao Teste de Student Newman Keuls $(\mathrm{P} \leq 0,05)$.

$\mathrm{O}$ parâmetro glicemia mensurado no T0 (basal) e no T60 (60 minutos) foi analisado com o Teste t pareado. Os escores referentes à sedação, ao relaxamento muscular e à resposta a estímulos foram avaliados pelo Teste não-paramétrico Kruskal-Wallis ANOVA por Ranks. Para os valores com diferença estatística, foi utilizado o Teste de Student Newman Keuls $(\mathrm{P} \leq 0,05)$ sendo que estes dados foram avaliados somente entre grupos.

\section{RESULTADOS}

Os resultados referentes aos parâmetros hemodinâmicos encontram-se dispostos na tabela 1 e figura 1. $\mathrm{Na}$ avaliação da $\mathrm{FC}$, foi observada diferença significativa entre grupos, com valores médios inferiores no GAX de T15 até T60 em comparação ao GA e GAD. Ocorreu redução na FC, no GAX e GAD, de T15 até T60 em comparação ao T0. Para o GA, houve redução de T30 até T60 quando comparado ao basal. Foi observada redução da PAS de T15 a T60, no GA e GAD, e apenas em T15 e T60 no GAX. Na avaliação entre grupos, os valores foram maiores no T30 e T45, no GAX. Para a PAM, houve redução em todos os grupos de T15 a T60. Em T45, os valores obtidos foram maiores no GAX em relação ao GA. Para a PAD, houve redução nos valores do T15 ao T60 nos três grupos.

Com relação a $\mathrm{SpO}$, o GAX apresentou valores menores no $\mathrm{T} 15$ em relação ao basal e em relação aos demais grupos. $\mathrm{A} \mathrm{PaCO}_{2}$ encontrou-se aumentada no GAX de T15 a T60 e valores de $\mathrm{PaO}_{2}$ significativamente diminuídos de T15 a T45 em relação ao T0, sem diferenças entre os grupos nesses parâmetros. O pH do GAD foi maior de T15 a T60 em relação ao T0. Para o $\mathrm{HCO}_{3}$, os valores de todos os tempos no GAX e GAD foram superiores em relação aos respectivos T0. Quanto ao EB, foram observados valores significativamente aumentados de T15 a T60 no GAD e de T30 a T60 no GAX em relação aos T0 correspondentes. Houve redução dos valores médios de $\mathrm{Hb}$ em todos os tempos do estudo, nos grupos GAD e GAX, com relação a T0.

Em relação aos escores de sedação obtidos no GAX, observou-se que $66,6 \%$ dos animais receberam escore 2 para sedação (moderada) e 33,4\% escore 1 (leve). Em relação ao relaxamento muscular, esse grupo obteve escore 2 em $66,6 \%$ dos animais e escore 3 (profundo) em 33,4\%. Nos grupos GA e GAD, todos os animais receberam escore 1 para sedação (leve), escore 0 (tônus normal) para relaxamento muscular em $83,4 \%$ dos animais e escore 1 (suave) em $16,7 \%$. Com relação à resposta aos estímulos sonoros, foram observados escores maiores de T15 até T45 no GAX, e 49,8\% dos animais desse grupo apresentaram escore $2 ; 33,4 \%$ escore 1 e $16,7 \%$ escore 0 .

Para os valores de $f, \mathrm{TR}, \mathrm{TC}, \mathrm{K}^{+}, \mathrm{Na}^{+}$e glicose não houve diferença significativa entre grupos ou entre os tempos dentro de cada grupo.

\section{DISCUSSÃO}

O maior grau de sedação, relaxamento muscular e abolição de resposta a estímulos, observados, no GAX, foi decorrente dos efeitos dosedependentes dos agonistas $\alpha_{2}$-adrenérgicos e da inibição do $\alpha$-adrenoceptor interneuronal no cordão espinhal (SINCLAIR, 2003). No entanto, segundo THURMON et al. (1996), a xilazina não promove potente efeito sedativo na espécie suína, apresentando sedação somente aparente, pois o animal responde à manipulação de maneira rápida. Da mesma forma, SAKAGUCHI et al. (1992) não obtiveram efeito analgésico com $2 \mathrm{mg} \mathrm{kg}^{-1}$ e GÓMEZ DE SEGURA et al. (1997) não observaram adequado efeito sedativo e analgésico com o emprego da xilazina em doses que variaram de $1 \mathrm{a} 16 \mathrm{mg} \mathrm{kg}^{-1}$. Nesse sentido, recomendase a utilização desse fármaco nessa espécie, associado a outros fármacos sedativos e/ou tranquilizantes (BAUCK, 1984). Assim, pode-se inferir que o maior efeito sedativo e miorrelaxante no GAX deve-se ao sinergismo produzido pelo azaperone, o qual, segundo COX (1973), quando administrado pela via IM nas doses de 1 a 2,5mg kg-1 , produz sedação e redução da agressividade; embora MUIR \& HUBBEL (2001) tenham afirmado que o relaxamento muscular não é propriedade característica dos tranquilizantes da classe das butirofenonas. Os resultados obtidos neste estudo para o GA reforçam os resultados relatados por MUIR 
Tabela 1 - Efeitos do azaperone e de sua associação com xilazina ou dexmedetomidina em suínos representação de médias e desvios-padrão em cada tempo do estudo.

\begin{tabular}{|c|c|c|c|c|c|c|}
\hline \multirow{2}{*}{ Variáveis } & \multirow{2}{*}{ Grupo } & \multicolumn{5}{|c|}{ 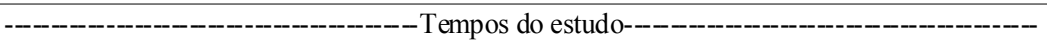 } \\
\hline & & T0 & $\mathrm{T} 15$ & $\mathrm{~T} 30$ & $\mathrm{~T} 45$ & $\mathrm{~T} 60$ \\
\hline \multirow{3}{*}{$\begin{array}{l}\text { FC } \\
\left(\text { bat } \min ^{-1}\right)\end{array}$} & GA & $146 \pm 7$ & $140 \pm 10$ & $127 \pm 15^{*}$ & $102 \pm 10^{*}$ & $119 \pm 4^{*}$ \\
\hline & GAD & $150 \pm 9$ & $128 \pm 11^{*}$ & $122 \pm 11 *$ & $113 \pm 8^{*}$ & $114 \pm 12^{*}$ \\
\hline & GAX & $146 \pm 12$ & $108 \pm 19^{*} \#$ & $99 \pm 10^{* \#}$ & $97 \pm 9^{* \#}$ & $99 \pm 11^{* \#}$ \\
\hline \multirow{3}{*}{$\begin{array}{l}\text { PAS } \\
(\mathrm{mmHg})\end{array}$} & GA & $110 \pm 14$ & $94 \pm 15^{*}$ & $87 \pm 7^{*}$ & $90 \pm 9^{*}$ & $93 \pm 2 *$ \\
\hline & $\mathrm{GAD}$ & $123 \pm 13$ & $101 \pm 10^{*}$ & $96 \pm 9^{*}$ & $94 \pm 8 *$ & $97 \pm 9 *$ \\
\hline & GAX & $117 \pm 6$ & $107 \pm 16^{*}$ & $111 \pm 12 \#$ & $110 \pm 14 \#$ & $107 \pm 15^{*}$ \\
\hline \multirow{3}{*}{$\begin{array}{l}\text { PAM } \\
(\mathrm{mmHg})\end{array}$} & GA & $94 \pm 10$ & $73 \pm 11$ & $67 \pm 8^{*}$ & $66 \pm 11 *$ & $72 \pm 6^{*}$ \\
\hline & GAD & $98 \pm 6$ & $82 \pm 8^{*}$ & $76 \pm 10^{*}$ & $75 \pm 4^{*}$ & $78 \pm 9 *$ \\
\hline & GAX & $99 \pm 6$ & $84 \pm 15^{*}$ & $83 \pm 13^{*}$ & $82 \pm 12 * \#$ & $83 \pm 12^{*}$ \\
\hline \multirow{3}{*}{$\begin{array}{l}\text { PAD } \\
(\mathrm{mmHg})\end{array}$} & GA & $80 \pm 13$ & $58 \pm 11^{*}$ & $53 \pm 9^{*}$ & $54 \pm 9^{*}$ & $58 \pm 7^{*}$ \\
\hline & GAD & $84 \pm 5$ & $71 \pm 6^{*}$ & $62 \pm 9^{*}$ & $62 \pm 6^{*}$ & $65 \pm 9 *$ \\
\hline & GAX & $86 \pm 7$ & $68 \pm 15^{*}$ & $68 \pm 11^{*}$ & $67 \pm 11 *$ & $70 \pm 11^{*}$ \\
\hline \multirow{3}{*}{$\begin{array}{l}f \\
\left(\operatorname{mov} \min ^{-1}\right)\end{array}$} & GA & $50 \pm 20$ & $50 \pm 15$ & $50 \pm 27$ & $50 \pm 35$ & $50 \pm 38$ \\
\hline & GAD & $46 \pm 12$ & $64 \pm 23$ & $43 \pm 17$ & $50 \pm 26$ & $46 \pm 21$ \\
\hline & GAX & $55 \pm 10$ & $48 \pm 21$ & $44 \pm 16$ & $44 \pm 16$ & $44 \pm 17$ \\
\hline \multirow{3}{*}{$\begin{array}{l}\mathrm{SpO}_{2} \\
(\%)\end{array}$} & GA & $96,4 \pm 0,5$ & $96,7 \pm 0,9$ & $96,4 \pm 0,8$ & $96,2 \pm 1,2$ & $96,7 \pm 0,3$ \\
\hline & GAD & $95,9 \pm 1,0$ & $96,4 \pm 0,5$ & $96,5 \pm 0,9$ & $96,1 \pm 0,8$ & $96,4 \pm 0,5$ \\
\hline & GAX & $96,6 \pm 0,3$ & $94,5 \pm 2,0 * \#$ & $95,4 \pm 0,7$ & $95,7 \pm 0,3$ & $95,8 \pm 1,3$ \\
\hline \multirow{3}{*}{$\begin{array}{l}\mathrm{PaO}_{2} \\
(\mathrm{mmHg})\end{array}$} & GA & 845 & $86 \pm 16$ & $86 \pm 9$ & $70 \pm 9$ & $84 \pm 5$ \\
\hline & GAD & $90 \pm 10$ & $86 \pm 5$ & $85 \pm 10$ & $72 \pm 9$ & $82 \pm 6$ \\
\hline & GAX & $89 \pm 6$ & $75 \pm 9 *$ & $77 \pm 6^{*}$ & $79 \pm 6^{*}$ & $81 \pm 13$ \\
\hline \multirow{3}{*}{$\begin{array}{l}\mathrm{PaCO}_{2} \\
(\mathrm{mmHg})\end{array}$} & GA & $34 \pm 6$ & $32 \pm 6$ & $35 \pm 5$ & $33 \pm 5$ & $34 \pm 3$ \\
\hline & GAD & $31 \pm 6$ & $33 \pm 4$ & $34 \pm 4$ & $35 \pm 2$ & $36 \pm 2$ \\
\hline & GAX & $32 \pm 5$ & $37 \pm 4 *$ & $38 \pm 3^{*}$ & $38 \pm 3^{*}$ & $39 \pm 3 *$ \\
\hline \multirow[t]{3}{*}{$\mathrm{pH}$} & GA & $7,47 \pm 0,02$ & $7,48 \pm 0,03$ & $7,48 \pm 0,02$ & $7,49 \pm 0,02$ & $7,50 \pm 0,01$ \\
\hline & GAD & $7,42 \pm 0,09$ & $7,48 \pm 0,02 *$ & $7,49 \pm 0,01^{*}$ & $7,50 \pm 0,01^{*}$ & $7,50 \pm 0,01^{*}$ \\
\hline & GAX & $7,45 \pm 0,05$ & $7,46 \pm 0,03$ & $7,47 \pm 0,01$ & $7,47 \pm 0,02$ & $7,48 \pm 0,01$ \\
\hline \multirow{3}{*}{$\begin{array}{l}\mathrm{HCO}_{3}^{-} \\
\left(\mathrm{mmolL}^{-1}\right)\end{array}$} & GA & $25,9 \pm 2,4$ & $25,5 \pm 3,6$ & $27,0 \pm 3,2$ & $22,9 \pm 4,1$ & $28,0 \pm 3,1$ \\
\hline & $\mathrm{GAD}$ & $22,5 \pm 5,8$ & $26,4 \pm 3,3$ & $26,5 \pm 3,2 *$ & $28,1 \pm 1,8^{*}$ & $29,2 \pm 1,7^{*}$ \\
\hline & GAX & $24,5 \pm 5,1$ & $26,8 \pm 3,4^{*}$ & $28,3 \pm 2,5^{*}$ & $28,9 \pm 2,8^{*}$ & $29,1 \pm 2,5^{*}$ \\
\hline \multirow{3}{*}{$\begin{array}{l}\text { EB } \\
\left(\mathrm{mmolL}^{-1}\right)\end{array}$} & GA & $1,5 \pm 2,7$ & $1,0 \pm 4,3$ & $2,8 \pm 3,7$ & $2,5 \pm 4,7$ & $3,9 \pm 3,5$ \\
\hline & GAD & $-2,5 \pm 7,3$ & $1,7 \pm 3,6^{*}$ & $2,8 \pm 3,6^{*}$ & $4,0 \pm 2,0^{*}$ & $5,2 \pm 1,7^{*}$ \\
\hline & GAX & $-0,1 \pm 6,0$ & $2,6 \pm 3,9$ & $4,2 \pm 2,7 *$ & $5,1 \pm 2,7^{*}$ & $5,1 \pm 2,7^{*}$ \\
\hline \multirow{3}{*}{$\begin{array}{l}\mathrm{Hb} \\
\left(\mathrm{g} \mathrm{dL}^{-1}\right)\end{array}$} & GA & $9,8 \pm 1,2$ & $9,1 \pm 1,1$ & $9,0 \pm 0,5$ & $8,4 \pm 0,5$ & $8,7 \pm 0,8$ \\
\hline & GAD & $10,8 \pm 1,4$ & $9,1 \pm 0,9^{*}$ & $8,7 \pm 1,0^{*}$ & $8,8 \pm 0,9^{*}$ & $9,3 \pm 1,1^{*}$ \\
\hline & GAX & $10,4 \pm 0,7$ & $9,3 \pm 0,8^{*}$ & $8,9 \pm 1,0^{*}$ & $8,7 \pm 0,7 *$ & $8,6 \pm 1,0^{*}$ \\
\hline
\end{tabular}

Tempos: T0 (basal); T1 5 (15 minutos após M0); T30 (30 minutos após M0); T45 (45 minutos após M0); T60 (60 minutos após T0).

Variáveis: frequência cardíaca (FC), pressão arterial sistólica, média e diastólica (PAS, PAM, PAD), frequência respiratória (f), saturação de oxihemoglobina $\left(\mathrm{SpO}_{2}\right)$, pressão parcial de oxigênio no sangue arterial $\left(\mathrm{PaO}_{2}\right)$, pressão parcial de dióxido de carbono no sangue arterial $\left(\mathrm{PaCO}_{2}\right)$, potencial de hidrogênio $(\mathrm{pH})$, bicarbonato de sódio $\left(\mathrm{HCO}^{-3}\right)$, excesso de base $(\mathrm{EB})$, hemoglobina $(\mathrm{Hb})$.

GA: grupo azaperone, GAD: grupo azaperone + dexmedet omidina, GAX: grupo azaperone + xilazina. * Difere de T0, Teste de Student Newman Keuls $(\mathrm{P}>0,05)$. \# Significativamente diferente dos demais grupos, Student Newman Keuls $(\mathrm{P}>0,05)$.

Ciência Rural, v.39, n.4, jul, 2009. 


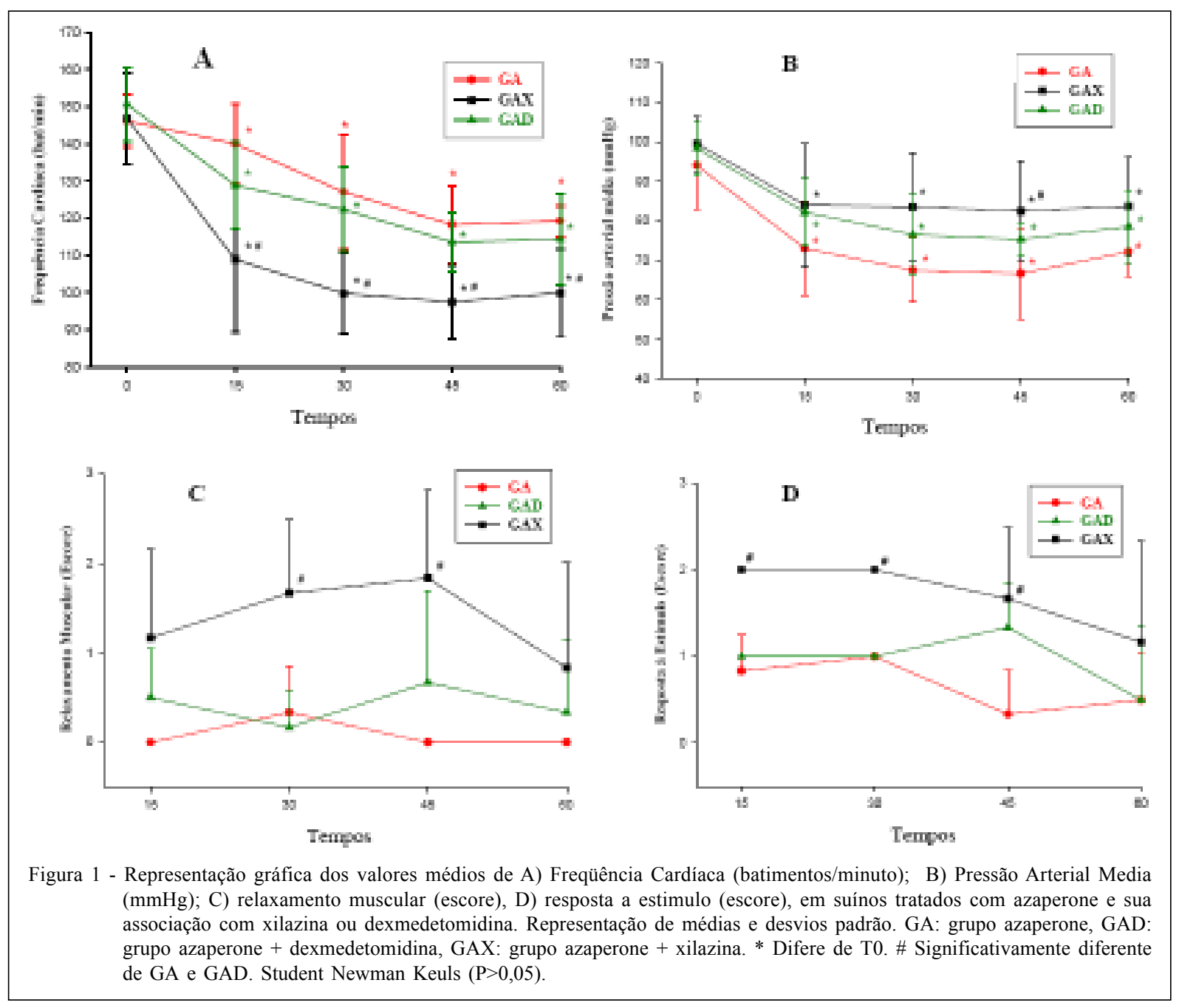

\& HUBBEL (2001), visto que foram observados pobre sedação e relaxamento muscular.

Em relação à dexmedetomidina, BAGATINI et al. (2002) e VILLELA et al. (2003), descreveram redução da concentração plasmática de catecolaminas em $90 \%$ associada com sedação, hipnose e analgesia, além da redução do requerimento de analgésicos no transoperatório. Porém, no presente estudo, não foi obtido efeito sedativo e miorrelaxante significativo quando esse fármaco foi utilizado associado ao azaperone, provavelmente em virtude das reduzidas doses utilizadas.

Observou-se redução significativa da FC no GAX em comparação aos demais grupos. Em todos os grupos houve redução entre tempos quando comparados ao T0. No entanto, todos os valores mantiveram-se dentro do limite fisiológico para a espécie. No GAX e GAD, tal redução decorreu do efeito vagomimético e da ação simpatolítica dos agonistas $\alpha_{2}$-adrenérgicos, os quais podem produzir intensa bradicardia especialmente em altas doses pela via IV (HAYASHI \& MAZE, 1993; KITAHARA et al., 2002). Reforçam esses resultados os achados de MOENS (2000), o qual afirma que, quando os agonistas $\alpha_{2}$ adrenérgicos são administrados pela via IM, produzem diminuição da FC menos acentuada do que quando administrados pela via IV. Da mesma maneira, GÓMEZ DE SEGURA et al. (1997), ao avaliarem os efeitos das doses de 1, 2, 4, 8 e $16 \mathrm{mg} \mathrm{kg}^{-1}$ de xilazina pela via IV em suínos, observaram bradicardia discreta com baixas doses e bradicardia moderada com o uso de altas doses. Por outro lado, a redução menos intensa da FC descrita no GAD está de acordo com os achados de VILLELA et al. (2003), os quais afirmam que a dexmedetomidina promove maior estabilidade hemodinâmica. Já a redução da FC observada no GA após 30 minutos da aplicação do fármaco coincide com o período de maior 
miorrelaxamento e sedação observado nos animais. Além disso os valores basais são maiores do que os considerados fisiológicos para animais adultos, devido ao estresse, conforme descrevem CENTENO et al. (2004). Cabe ressaltar que os animais utilizados no presente estudo possuíam 50 dias de idade, o que justifica os valores maiores encontrados no T0. Conforme SHORT (1987), o grau de depressão cardiopulmonar do azaperone é dose-dependente e comumente seriam observados hipotensão e taquicardia reflexa, fato não observado no GA devido provavelmente à baixa dose empregada. Por outro lado, ADETUNJI \& AJAO (2001) relataram estimulação cardiorrespiratória após administração de $8 \mathrm{mg} . \mathrm{kg}^{-1} \mathrm{de}$ azaperone pela via IM sem causa provável para este efeito, mas relataram que pode ser decorrente da severa hipotensão promovida pelo azaperone.

Observou-se hipotensão em todos os grupos, pois, de acordo com CENTENO et al. (2004), os valores normais para PAS e PAD são 100 e $60 \pm 10 \mathrm{mmHg}$, respectivamente, para o suíno adulto. No GA, esse fato decorre do efeito do azaperone sobre a musculatura lisa vascular, promovendo diminuição na resistência vascular sistêmica mediada pelo bloqueio a-adrenérgico (SAKIHARA et al., 1996). Da mesma forma, VAN WOERKENS et al. (1990) observaram redução de $35 \%$ na pressão arterial e de $10 \%$ no débito cardíaco, 15 minutos após a administração de $5 \mathrm{mg} \mathrm{kg}^{-1}$ de azaperone pela via IM em suínos adultos.

Para o GAX e GAD, observou-se hipotensão menos acentuada, não evidenciando-se efeito bifásico, ou seja, hipertensão seguida por hipotensão como sugerido por BAGATINI et al. (2002). Esse fato pode ser explicado pela via de administração (IM) e pelas doses reduzidas utilizadas neste estudo. Além disso, a primeira avaliação ocorreu 15 minutos após a aplicação dos fármacos, e a hipertensão pode ter ocorrido antes da primeira mensuração. Reforçam esses achados os resultados obtidos por FLÔRES et al. (2008) os quais demonstraram que a dexmedetomidina, na dose de $3 \mu \mathrm{g} \mathrm{kg}^{-1}$, pela via IV, em cães, produziu hipertensão arterial severa durante os 60 minutos de avaliação. Por outro lado, GÓMEZ DE SEGURA et al. (1997) ao avaliarem os efeitos das doses de $1,2,4,8$ e $16 \mathrm{mg} \mathrm{kg}^{-1}$ de xilazina via IV em suínos, observaram bradicardia e efeito bifásico da pressão arterial com hipertensão inicial seguida de hipotensão com o emprego de baixas doses. Da mesma forma, MOENS (2000) relatou que a xilazina, quando administrada via IV, induz curto período de hipertensão (cinco a 10 minutos) seguido de longa hipotensão.

Não foram observadas diferenças significativas para a $f$ em nenhum momento dos grupos estudados. Esses resultados assemelham-se aos descritos por SAKAGUCHI et al. (1992), os quais empregaram $2 \mathrm{mg} \mathrm{kg}^{-1}$ de xilazina em suínos, HENRIKSON et al. (1995), os quais utilizaram azaperone, e VILLELA et al. (2003), que avaliaram o uso de dexmedetomidina.

A saturação de oxihemoglobina periférica $\left(\mathrm{SpO}_{2}\right)$ foi menor somente no GAX em T15. Isso ocorreu devido à depressão respiratória produzida pela xilazina caracterizada não pela redução da $f$, mas pela redução da amplitude respiratória, fato justificado pelos menores valores de $\mathrm{PaO}_{2}$ e maiores de $\mathrm{PaCO}_{2}$ a partir desse momento até T60 em relação a T0. Da mesma forma MOENS (2000), observou redução da $\mathrm{PaO}_{2}$ e acréscimo da $\mathrm{PaCO}_{2}$ com doses clínicas de xilazina, devido à localização de $\alpha_{2}$-adrenoceptores periféricos e a um desequilíbrio entre a perfusão relacionada à ventilação e circulação pulmonar.

Quanto à TR, apesar de os agentes butirofenônicos produzirem vasodilatação periférica (MUIR \& HUBBEL, 2001), não ocorreram alterações significativas durante o estudo, fato auxiliado pelo controle de temperatura da sala, que foi mantido em torno de $23,5^{\circ} \mathrm{C}$, ao contrário dos achados de SAKAGUCHI et al. (1992), os quais observaram hipotermia após a utilização da mesma dose de xilazina em suínos, em ambiente não climatizado.

O aumento significativo no GAD dos valores de $\mathrm{pH}$, e dos valores de $\mathrm{HCO}_{3}^{-}$e EB em GAX e GAD, evidenciam a ocorrência de alcalose metabólica. Avaliando os dados obtidos, verifica-se que os animais estudados já apresentavam alcalose metabólica e respiratória desde o período basal, o que caracteriza um distúrbio ácido básico misto (MORAES \& LEISEWITZ, 2007), que pode ser causado por taquipnéia de estresse inicial decorrente da manipulação ou mesmo por dietas pobres em cloretos, produzindo elevação de $\mathrm{HCO}_{3}^{-} \mathrm{e}$ pH. Essa hipótese não pôde ser comprovada neste estudo, pois o íon cloro não foi avaliado.

A redução da $\mathrm{Hb}$ observada no GAX e GAD pode ser explicada pelo efeito adrenolítico do azaperone sobre o baço. Em animais conscientes, as catecolaminas respondem ao estresse por meio de contração esplênica levando ao aumento de hemoglobina. Essa resposta do baço é minimizada com o emprego de fármacos sedativos ou associações com propriedades adrenolíticas, induzindo uma redução dos valores de $\mathrm{Hb}$ (ADETUNJI \& OSUNBUNMI., 2000).

\section{CONCLUSÃO}

De acordo com os resultados obtidos, conclui-se que a associação de azaperone e xilazina nas doses propostas, produziu maior sedação e miorrelaxamento e menor resposta aos estímulos. 
Estudos complementares são necessários para confirmar que o azaperone, associado aos fármacos agonistas $\alpha_{2}$-adrenérgicos, ameniza a hipertensão produzida por esses.

\section{COMISSÃO DE ÉTICA}

O presente estudo foi aprovado pela Comissão de Ética e do Bem-Estar Animal da Universidade do Estado de Santa Catarina, conforme protocolo 129/04.

\section{FONTES DE AQUISIÇÃO}

a-Isoforine: Cristália Produtos Químicos e Farmacêuticos Ltda. Itapira, SP, Brasil.

b-BD PRN Adapter - Becton, Dickinson de México, S.A. de C.V., México.

c-Suicalm - Azaperone 40mg/ml. Janssen Pharmaceutica. Beerse, Bélgica.

d-Precedex - Cloridrato de Dexmedetomidina. 100 $\mu \mathrm{g} / \mathrm{ml}$. Abbott Ltda. RJ, Brasil.

e. Sedazine - Cloridrato de Xilazina 10\%. Fort Dodge Inc. SP, Brasil.

f-Spacelabs Medical Multiparamétrico 90496 - USA.

g-Accu-chek Advantage II - Roche S/A. São Paulo, SP, Brasil.

\section{REFERÊNCIAS}

ADETUNJI, A.; OSUNBUNMI, O. Haematological effect of azaperone sedation in pigs. Afric J Biom Researc v.3, n.2, p.131-133, 2000 .

ADETUNJI, A.; AJAO, A.O. Comparison of extradural injections of lignocaine and xylazine in azaperone-sedated pigs. Veterinary Journal, v.161, p.98-99, 2001. Disponível em: $<\mathrm{ht} \mathrm{t} \mathrm{p} \mathrm{:} \mathrm{/} \mathrm{/} \mathrm{w} \mathrm{w} \mathrm{w.} \mathrm{s} \mathrm{c} \mathrm{i} \mathrm{e} \mathrm{n} \mathrm{c} \mathrm{ed} \mathrm{i} \mathrm{r} \mathrm{e} \mathrm{t.} \mathrm{c} \mathrm{o} \mathrm{m/}$ science? ob=ArticleURL\& udi=B6WXN-457C8T8K\&_user $=687358 \&$ \&doc $=1 \&$ fmt $=\&$ \&orig $=$ search $\&$ _sort $=\mathrm{d}$ $\& \mathrm{vi}$ e w $=\mathrm{c} \&$ a c c t $=$ C $000037899 \&$ version $=1 \&$ u r 1 V e r s i o $n=0 \&$ u s e r $\overline{\mathrm{i}} \mathrm{d}=68735$ $8 \&$ md $5=$ cfc9e4204aa2e8a69029bee0df6b4be9>. Doi: $10.1053 /$ tvj1.2000.0518.

BAGATINI, A. et al. Dexmedetomidina: farmacologia e uso clínico. Rev Bras Anestesiol, v.52, n.5, p.606-617, 2002. Disponível em: $<$ http://www.scielo.br/scielo.php?script $=$ sci arttext\&pid $=$ S0034 $70942002000500012 \& \operatorname{lng}=$ en $\&$ nrm $=$ iso\&tlng $=$ pt $>$. Doi: $10.1590 /$ S0034-70942002000500012.

BAUCK, S.W. An evaluation of a combination of injectable anesthetic agents for use in pigs. Can Vet J, v. 25, p.162-165, 1984.

CENTENO, M.A.L. et al. Cambios fisiológicos em cerdo de cirurgia experimental para transplante cardíaco. Investigación em Salud, v.6, n.1, p.11-13, 2004.

COX, J.E. Immobilization and anaesthesia of the pig. Vet Rec, v.92, p.143-147, 1973.

FLÔRES, F.N. et al. O sulfato de atropina nos parâmetros hemodinâmicos e hemogasométricos de cães anestesiados com clorpromazina, dexmedetomidina e isofluorano. Ciência Rural, v.38, n.4, p.1024-1029, 2008 .

GÓMEZ DE SEGURA, I.A. et al. Actions of xylazine in young swine. Am J Vet Res, v.58, n.1, p.99-102, 1997.
HAYASHI, Y.; MAZE, M. Alpha ${ }_{2}$ adrenoreceptor agonist and anesthesia. Br J Anaesth, v.71, p.108-118, 1993.

HENRIKSON, By H. et al. Anaesthetics for general anaesthesia in growing pigs. ActaVet Scand, v.36, p.401-411, 1995.

KITAHARA, F.R. et al. Efeitos hemodinâmicos da dexmedetomidina em cães. Estudo experimental. R Bras Ci Vet, v.9, n.1, p.128-130, 2002.

MOENS, Y. The veterinary experience. Bailleiere Clin Anaesth, v.14, n.2, p.293-304, 2000.

MORAES, H.A.; LEISEWITZ, A.L. Distúrbios ácido-básicos mistos. In: DIBARTOLA, S.P. Anormalidades de fluídos, eletrólitos e equilíbrio ácido-básico na clínica de pequenos animais. 3.ed. São Paulo: Roca, 2007. Cap.12, p. 283-294.

MUIR, W.W.; HUBBELL, J.A.E. Manual de anestesia veterinária. 3.ed. Porto Alegre: Artmed, 2001. 432p.

ORR, J.A. et al. Cardiopulmonary effects of the combination of neuroleptic azaperone and hypnotic metomidate in swine. Am J Vet Res, v.37, n.11, p.1305-1308, 1976.

SAKAGUCHI, M. et al. Sedative effects of medetomidine in pigs. J Vet Med Sci, v.54, n.4, p.643-647, 1992.

SAKIHARA, C. et al. Direct inhibitory effect of chlorpromazine on smooth muscle of the porcine pulmonary artery. Anesthesiol, v.85, n.3, p.616-625, 1996.

SAVOLA, N.K.T.; SAVOLA, J.M. Dexmedetomidine, an $\alpha_{2}$ adrenoceptor agonist, detects a novel imidazole binding site in adult rat spinal cord. European J Pharmalogy, v.306, p.315323,1996

SHORT, C.E. Principles and practice of veterinary anesthesia Veterinary. Baltimore: Williams \& Wilkins, 1987. 669p.

SINCLAIR, M.D. A review of the physiological effects of $\alpha_{2}$ agonists related to the clinical use of medetomidine in small animal practice. Can Vet J, v.44, p.885-896, 2003.

TAKROURI, M.S. et al. Dexmedetomidine in intensive care unit: a study of hemodynamic changes. Middle East J Anesthesiol, v.16, n.6, p.587-595, 2002.

THURMON, J.C. et al. Lumb \& Jones: veterinary anesthesia. 3.ed. Baltimore: Williams \& Wilkins, 1996. 1132p.

TRANQUILLI, W.J.; GRIMM, K.A. Pharmacology of drugs used for anesthesia and sedation. Vet Clin North Am: Food Anim Pract, v.12, n.3, p.501-523, 1996.

VAN WOERKENS, L.J. et al. Redistribution of cardiac output caused by opening of arteriovenous anatomoses by a combination of azaperone and metomidate. Br J Anaesth v.65, n.3, p.393-399, 1990 .

VILLELA, N.R. et al. Efeitos cardiovasculares de duas doses de dexmedetomidina. Estudo experimental cães. Rev Bras Anestesiol, v.53, n.6, p.784-796, 2003. Disponível em: <http:// www.scielo.br/scielo.php? script $=$ sci arttext\&pid $=$ S0034$70942003000600009 \& \operatorname{lng}=$ en\&nrm $=$ iso\&tlng $=p t>$. Doi: $10.1590 /$ S0034-70942003000600009. 